\title{
A comparison of HDL-C, VLDL-C among diabetics with and without dyslipidemia.
}

1. MBBS, M.Phil

Assistant Professor Biochemistry

Foundation University Medical

College, Islamabad.

2. MBBS, M.Phil, PhD.

Associate Professor Biochemistry and Molecular Biology

Army Medical College,

National University of Medical

Sciences

Abid Majeed Road, Rawalpindi.

3. M.Phil, PhD., Post Doc

Associate Professor Biochemistry and Molecular Biology

Army Medical College,

National University of Medical

Sciences

Abid Majeed Road, Rawalpindi.

Correspondence Address:

Dr. Hajira Siddique

Department of Biochemistry

Foundation University Medical

College, Islamabad.

shaheer1101@gmail.com

Article received on:

23/10/2019

Accepted for publication:

25/02/2020

\begin{abstract}
Hajira Siddique', Sarah Sadiq ${ }^{2}$, Asifa Majeed ${ }^{3}$
\end{abstract}
ABSTRACT... Objectives: To determine and compare the levels of HDL-C and VLDL-c between Diabetic with/without dyslipidemia with normal healthy controls and risk of Cardiovascular disease. Study Design: Cross Sectional Comparative study. Setting: Multidisciplinary Lab I, Department of Biochemistry and Molecular Biology Army Medical College, Rawalpindi. Period: 2 years January 2016 to January 2018. Material \& Methods: Total 90 subjects were enrolled in three groups i.e., group I comprised of 30 patients of Diabetic dyslipidemia, group II consisted of 30 patients of Diabetes without dyslipidemia and group III consisted of 30 healthy normal controls. Demographic and clinical data were collected. Data collected was analyzed by SPSS version 22. Results: Male to female ratio included in group I was 1:1.73 and in group II and III was 1:1.5 each. Mean value of the HDL-c among group I was significantly lower as compared to controls. Mean values of the triglycerides (TG) among group I and group II were significantly elevated as compared to the controls. Most of the subjects of group III were doing exercise as part of everyday routine. Conclusion: The HDL-C and TG (VLDL-C) levels are perturbed significantly among Diabetic dyslipidemic patients as compared to Diabetic non-dyslipidemic patients and normal healthy controls. Exercise is an important factor missed by patients to manage their disease.

Key words: Dyslipidemia, HDL-c, Type 2 Diabetes Mellitus, VLDL-c.

Article Citation: Siddique H, Sadiq S, Majeed A. A comparison of HDL-C, VLDL-C among diabetics with and without dyslipidemia. Professional Med J 2020; 27(11):2427-2432. https://doi.org/10.29309/TPMJ/2020.27.11.4282

\section{INTRODUCTION}

Non communicable diseases (NCDs), also referred to as chronic diseases, mainly include 4 types i.e., cardiovascular diseases, cancers, respiratory diseases and diabetes which together are responsible for more than $80 \%$ of all early NCD deaths. ${ }^{1}$ Cardiovascular diseases are ranked $1^{\text {st }}$ among NCDs with highest death rate i.e., 17.7 million people per year and diabetes ranked $4^{\text {th }}$ causing 1.6 million deaths annually. ${ }^{2}$ NCDs predominantly affect the population of lowas well as middle-income countries. ${ }^{3}$

Epidemic surge in the victims of diabetes particularly type 2 diabetes has been observed for the past three decades, and predominantly in developing countries, where $>80 \%$ of the population are suffering from diabetes. It is assessed that, in South Asia the prevalence of type 2 Diabetes is $>150 \%$ amid 2000 and $2035 .{ }^{4}$
According to International Diabetes Federation (IDF), as of 2015, there were more than 7 million Diabetes patients in Pakistan. ${ }^{5}$

Diabetes Mellitus (DM) is hyperglycemic condition caused by in-sufficient production/ action of insulin. In chronic cases, DM leads to damage, dysfunction, and failure of different organs. ${ }^{6}$ Diabetes is accompanied by numerous complications. Most of the patients suffering from both type 1 and type 2 forms of diabetes encounter these complications. ${ }^{7}$ The diabetes associated complications are divided into Microvascular disease and macrovascular disease. Microvascular disease is caused by damage to small blood vessels whereas macrovascular disease is the result of damage to the arteries. Microvascular complications refers to retinopathy, nephropathy and neuropathy whereas major macrovascular complications associated with 
diabetes accelerate cardiovascular disease that leads to myocardial infarction (heart attack) and cerebrovascular disease (stroke). ${ }^{7}$

As DM is linked with a significantly higher risk of cardiovascular disease, dyslipidemia is also one of the common complications among diabetic patients. ${ }^{8}$ Although diagnosis of diabetes is based on raised glucose levels, appearance of dyslipidemia is also witnessed in such patients which is defined by raised low density lipoprotein (LDL-C) cholesterol and triglyceride (TG) concentrations, and diminished concentration of high density lipoprotein (HDL-C) cholesterol in the blood. ${ }^{9}$ Hyperglycemia and insulin resistance in type 2 DM plays a dominant part in development of Diabetic Dyslipidemia and is linked with significantly increased production of triglyceride rich lipoproteins TRL harboring surface apoB including very low-density lipoprotein (VLDL) and chylomicrons. ${ }^{10}$ Diabetic dyslipidemia, i.e. high triglycerides (TG) and low HDL cholesterol (HDL-C) are considered one of the chief cardiovascular disease (CVD) risk factors and even considered as an equivalent to myocardial infarction. ${ }^{11}$ Presence of T2DM and dyslipidemia both increase the $\mathrm{CV}$ risk by 3-4 times compared to non-diabetic patients with dyslipidemia. ${ }^{12}$ Strong evidence indicates that cardiovascular outcomes can be improved by lowering cholesterol levels. ${ }^{8}$

The pathways underlining diabetic complications with protective or pathological role still remain elusive. Moreover, apparently independent pathways are also significantly interacting with each other to aggravate pathology. Dyslipidemia is also thought to be a factor aggravating these manifestations. ${ }^{7}$ This led us to assess some clinical manifestations of the diabetes and dyslipidemia. For this purpose, the study was designed to evaluate the levels of HDL-C and VLDL-C in diabetic patients with and without dyslipidemia and their potential roles in cardiovascular complications associated with diabetes among Pakistani subjects.

\section{Objective of the study was to}

- Assess the overall levels of HDL-C and VLDL-C in Diabetic patients, Diabetic patients with dyslipidemia and normal healthy controls.

- Comparing the levels of HDL-C and VLDL-C between diabetic with/without dyslipidemia by comparing with normal healthy controls and risk of cardiovascular disease.

- To evaluate the HDL-C and VLDL-C levels as marker to determine the risk of cardiovascular diseases.

HDL-C and VLDL-C levels are hypothesized to be elevated among diabetic dyslipidemic patients as compared to diabetic and normal healthy subjects and can be used as markers to determine the risk of cardiovascular diseases.

\section{MATERIAL \& METHODS}

The research was conducted after the formal approval of AMC's Ethical Review Committee. For this purpose, the first step was to obtain informed written consent of all the participants included in our research. The patients having incomplete data were excluded from the final data analysis. The patients included were also kept informed about the outcomes of the study. In order to make sure the secrecy, each patient was assigned a code and was analyzed based on that code.

The study design for research was crosssectional comparative based. The study was conducted at multidisciplinary lab 1, department of Biochemistry and Molecular biology Army Medical College, Rawalpindi.

Total 90 subjects were enrolled in the study using convenient sampling technique and divided in three groups.

Group I comprised of 30 patients of diabetics with dyslipidemia complication

Group II comprised of 30 patients of known diabetics without dyslipidemia

Group III comprised of 30 healthy normal controls.

The participants of all the three groups were assessed for:

1. Gender difference/ gender ratio

2. HDL-c assessment

3. VLDL-c assessment

The group included type 2 DM patients excluding 
type 1 DM affected patients. The patients on lipid lowering drugs were also excluded from the study.

The participants of each group were analyzed for gender ratio. The gender ratio was then compared among the three groups.

Blood samples were obtained under sterile conditions from all 60 participants distributed among three designated groups visiting Fauji foundation Hospital, Rawalpindi. Blood sample was taken from median cubital vein and saved in specimen tubes with proper identification code for each individual. The blood samples were then processed for HDL-C and VLDL-C assessment. HDL-C levels among the subjects of the study were analyzed using the procedure of the HDLDirect kit by in noline merck (5.17518.0001). The normal range of the HDL-C was> 1.0expressed in units $\mathrm{mmol} / \mathrm{l}$. VLDL-C levels among the subjects of the study were analyzed using the formula VLDL $=$ triglycerides $(\mathrm{mmol} / \mathrm{L}) / 2.2$ used..$^{12} \mathrm{TGs}$ were measured by Diasys triglycerides FS (Cat \# 157109910021). The normal range of the VLDL-C was $<1.04$ expressed in unit's $\mathrm{mmol} / \mathrm{l}$.

Data was collected and analyzed using SPSS Version 22. Categorical data was presented in the form of percentages. Comparison of variables among groups was done using chi-square and/ or Student's t-test as applicable. The values at 95\% confidence interval $(\mathrm{Cl})$ were considered as significant.

\section{RESULTS}

Among group I subjects, 11 (36.67\%) patients were male whereas 19 (63.33\%) patients were female making the male to female ratio as 1:1.73. Among group II subjects, 12 (40\%) patients were male whereas 18 (60\%) patients were female with ratio being 1:1.5. Same ratio was also observed among subjects of the group III i.e., normal healthy controls where $12(40 \%)$ of the 30 subjects were male ad 18 (60\%) were female. The statistical analysis showed that there was no significant difference among the groups with respect to gender ratio $(p=0.954)$ as shown in Figure-1.
Mean values of the HDL-c were calculated along with their standard deviations for all the three groups. The mean HDL-c value of the group 1 subjects i.e., diabetic patients with dyslipidemia was found to be $0.86( \pm 0.09)$. The mean HDL-C value of the group II subjects i.e., diabetic patients without dyslipidemia was found to be higher (1.11 $\pm 0.16)$ as compared to group I. The mean HDL-c value of the group III subjects i.e., normal healthy participants was found to be highest $(1.18 \pm 0.23)$. The statistical analysis showed that there was highly significant difference between the groups i.e., $p<0.001$ between group I and group II and $p$ $<0.001$ between group I and group III whereas no significant difference was found between group 2 and group $3(p=0.108)$ (Table-I).

Mean values of the triglycerides were also calculated along with their standard deviations for all the three groups. The mean TG (VLDL) value of the group I subjects i.e., diabetic patients with dyslipidemia was found to be 2.45 ( \pm 0.57$)$. The mean TG (VLDL) value of the group II subjects i.e., diabetic patients without dyslipidemia was found to be lower $(1.51 \pm 0.23)$ as compared to group I. The mean TG (VLDL) value of the group III subjects i.e., normal healthy participants was found to be lowest $(2.45 \pm 0.57)$. The statistical analysis showed that there was highly significant difference between the groups i.e., $p<0.001$ between group I and group II, $p<0.001$ between group I and group III and $p=0.009$ between group II and group III.

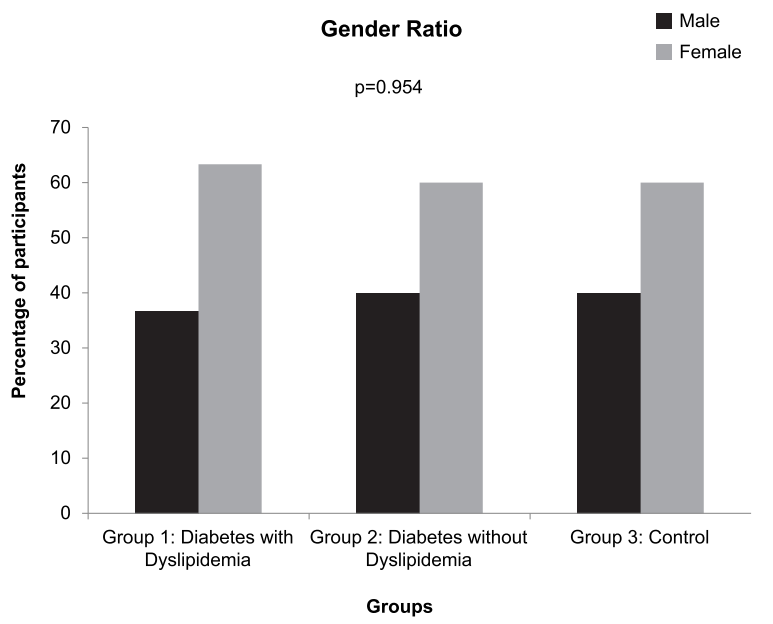

Figure-1. Graphical representation of the gender ratio between different groups 
Among group I subjects, almost all subjects i.e., 29 (96.67\%) patients went through exercises/ physical activity infrequently whereas only 1 patient (3.33\%) was exercising regularly. Among group II subjects, 2 patients (6.67\%) were not doing any kind of exercise as part of their everyday life, 27 patients (90\%) went through exercises/ physical activity infrequently whereas only 1 patient $(3.33 \%)$ was exercising regularly. Among group III subjects, 17 healthy subjects (56.67\%) were not doing any kind of exercise as part of their everyday life whereas 13 subjects (43.33\%) were doing regular exercises/ physical activity. Comparing the number of subjects doing regular exercise with those who are not doing it irregularly showed a statistical significant difference among the three groups $(p<0.001)$.

\begin{tabular}{|l|c|c|c|c|}
\hline \multicolumn{1}{|c|}{ Groups } & Average & SD & \multicolumn{2}{c|}{ Comparison(p value) } \\
\hline Group 1: Diabetes with Dyslipidemia & 0.86 & 0.09 & Group 1 vs Group 2 & $<0.001$ \\
\hline Group 2: Diabetes without Dyslipidemia & 1.11 & 0.16 & Group 1 vs Group 3 & $<0.001$ \\
\hline Group 3: Control & 1.18 & 0.23 & Group 2 vs Group 3 & 0.108 \\
\hline
\end{tabular}

Table-I. Tabular representation of the HDL-c assessment and comparison between different groups

\section{DISCUSSION}

The connection between lipid profile disturbance and diabetes related complications has long been given attention. ${ }^{13}$ Dyslipidemia is a strong predictor of cardiovascular disease and deaths in diabetic patients. ${ }^{13}$ Diabetic dyslipidemia is marked by high levels of plasma TG and low levels of HDL-C. ${ }^{14,15}$ Thus keeping in view the link between diabetes and dysplasia with cardiovascular disease, we examined the HDL-C and TG levels in diabetic patients with dyslipidemia as compared to normal healthy controls. But in order to get an insight into the pattern of TG and HDL-C levels in diabetic non dyslipidemic patients, we conducted a little broader study including three groups i.e., the diabetic dyslipidemic patients, diabetic nondyslipidemic patients and normal healthy controls. The results showed that TG (VLDL-C) levels were significantly elevated in diabetic patients (both with and without dyslipidemia) as compared to the normal healthy controls. The TG levels were also significantly different among the diabetic patients with elevated levels among diabetic dyslipidemia patients as compared to diabetic patients without dyslipidemia. At the same time, significantly lower levels of HDL-C were observed between the diabetic patients with dyslipidemia complications as compared to the normal healthy controls. HDL-C levels were significantly lower among diabetic dyslipidemic patients as compared to diabetic non-dyslipidemic patients. The disturbed levels of TG and HDL-C seem to root from diabetes without dyslipidemia and ultimately lead to dyslipidemia in diabetic patients. Hence elevation of HDL-C and VLDL-C levels can be used as marker to determine the risk of diabetes, diabetic dyslipidemia and ultimately cardiovascular diseases.

There are number of studies that focused to investigate TG and HDL-C levels among patients with diabetic dyslipidemia. A study was conducted on 576 patients including 192 normal healthy controls, 192 diabetic subjects with atherosclerosis and 192 non-diabetic atherosclerotic patients. All the subjects were analyzed for biochemical parameters i.e., fasting blood sugar levels and lipid constituents including triglycerides (TG) and high-density lipoprotein (HDL) levels. The results showed that diabetic atherosclerotic patients had elevated TG level and low HDL level as compared to non-diabetic atherosclerotic patients and normal healthy controls. ${ }^{13}$ Another study was conducted to assess the epidemiology, treatment, and management of dyslipidemia among diabetic patients in USA. The analysis of HDL-C and TG levels showed that HDL-C levels were low in more than half of males and more than two-thirds of females. More than half were found to have high elevated TG levels. Dyslipidemia remained uncontrolled for many diabetic patients. ${ }^{14}$

For years, exercise is considered to be basis for diabetes management in addition to diet and medication. But until recently, the ample evidence regarding importance of exercise/physical activity 
and fitness in diabetes was missing. ${ }^{16}$ T2DM is getting common mainly due to increasing adoption of sedentary way of life and obesity. The effect of changes in life style including physical activity on preventing T2DM among high risk subjects were evaluated in a study. The results showed that risk of acquiring diabetes was significantly reduced i.e., 58\% $(p<0.001)$ through changes in lifestyle including exercise (30 min per day). It was also found that exercising more than $4 \mathrm{hrs} /$ week significantly reduced the risk of getting diabetes in subjects without weight loss. Any form of physical activity like sports, gardening, household activity etc can have same benefits in preventing diabetes. ${ }^{17}$ The findings are in harmony with our observations. We have also observed in our study that patients with diabetes including both with and without dyslipidemia were going through infrequent exercise as part of their everyday life. The control group (normal healthy people) has significantly higher percentage of subjects doing regular exercise. Hence exercise is among the most important factor to keep the person fit and healthy and should be included in everyday routine. Exercise with proper guidance can reduce the risk of diabetes but at the same time improper/irregular exercise patterns can deteriorate the situation.

The study directs conclusive recommendations for doctors, patients as well as normal healthy subjects. The doctors are advised to regularly go through lipid profile of the diabetic patients so that they can be prevented from acquiring diabetes associated complications. The diabetic patients are advised to take care of their sugar levels, go for regular medical checkups, focus on diet plan and perform physical activity/ exercise to ensure good health and improved quality of life. They should regularly monitor their HDL-C and TG levels as their perturbed levels lead to dyslipidemia and ultimately cardiovascular diseases (heart attack and strokes). The normal healthy subjects should go through regular blood tests to monitor and timely treat slight disruption in lipid profiles and other biochemical parameters. Exercise should be scheduled at least 30min per day as part of their everyday routine for their health and fitness. Hence regular checkups, change in life style including exercise can reduce the risk and impact of different diseases and increases the quality of life and life span.

\section{CONCLUSION}

The HDL-C and TG (VLDL-C) levels are perturbed significantly among diabetic dyslipidemic patients as compared to diabetic non-dyslipidemic patients and normal healthy controls. HDL-C and VLDL-C assessment can be used as markers to determine the risk of cardiovascular diseases. Exercise is an important factor missed by patients to manage their disease.

\section{Copyright $@ 25$ Feb, 2020.}

\section{REFERENCES}

1. Nugent RA, Husain MJ. Introducing the PLOS special collection of economic cases for NCD prevention and control: A global perspective. 2020; 15(2):e0228564.

2. NCD Countdown 2030: Worldwide trends in noncommunicable disease mortality and progress towards Sustainable Development Goal target 3.4. Lancet (London, England). 2018; 392(10152):1072-88.

3. Organization. WH. No communicable diseases. Fact sheet Updated June 2017. Available from: http://www. who.int/mediacentre/factsheets/fs355/en/.

4. Nanditha A, Ma RC, Ramachandran A, Snehalatha C, Chan JC, Chia KS, et al. Diabetes in Asia and the Pacific: Implications for the Global Epidemic. Diabetes care. 2016; 39(3):472-85.

5. Freeman S, Eddy SL, McDonough M, Smith MK, Okoroafor $\mathrm{N}$, Jordt $\mathrm{H}$, et al. Active learning increases student performance in science, engineering, and mathematics. Proc Natl Acad Sci U S A. 2014; 111(23):8410-5.

6. Alam U, Asghar O, Azmi S, Malik RA. General aspects of diabetes mellitus. Handbook of clinical neurology. 2014; 126:211-22.

7. Forbes JM, Cooper ME. Mechanisms of diabetic complications. Physiological reviews. 2013; 93(1):13788.

8. Schofield JD, Liu Y, Rao-Balakrishna P, Malik RA, Soran H. Diabetes dyslipidemia. Diabetes therapy: Research, treatment and education of diabetes and related disorders. 2016; 7(2):203-19.

9. Duval C, Muller M, Kersten S. PPAR alpha and dyslipidemia. Biochimica et biophysica acta. 2007; 1771(8):961-71. 
10. SzalatA, DurstR, LeitersdorfE. Managing dyslipidaemia in type 2 diabetes mellitus. Best practice \& research Clinical endocrinology \& metabolism. 2016; 30(3):43144.

11. Closs Cl, Ruiz Diaz MA, Cafferata AM, Becu-Villalobos D, Nogueira JP. [Role of the enterocyte in type 2 diabetes mellitus associated dyslipidemia]. Medicina. 2018; 78(2):91-8.

12. Martins J, Olorunju SA, Murray LM, Pillay TS. Comparison of equations for the calculation of LDLcholesterol in hospitalized patients. Clinica chimica acta; international journal of clinical chemistry. 2015; 444:137-42.

13. Ali F, Jamil H, Anwar SS, Wajid N. Characterization of lipid parameters in diabetic and non-diabetic atherosclerotic patients. Journal of geriatric cardiology: JGC. 2015; 12(1):37-43.
14. Mooradian AD. Dyslipidemia in type 2 diabetes mellitus. Nature clinical practice Endocrinology \& metabolism. 2009; 5(3):150-9.

15. Jacobs MJ, Kleisli T, Pio JR, Malik S, L'Italien GJ, Chen $\mathrm{RS}$, et al. Prevalence and control of dyslipidemia among persons with diabetes in the United States. Diabetes research and clinical practice. 2005; 70(3):2639.

16. Sigal RJ, Kenny GP, Wasserman DH, CastanedaSceppa C, White RD. Physical activity/exercise and type 2 diabetes: A consensus statement from the American Diabetes Association. Diabetes care. 2006; 29(6):1433-8.

17. Tuomilehto J, Lindstrom J, Eriksson JG, Valle TT, Hamalainen $H$, llanne- $P$ arikka $P$, et al. Prevention of type 2 diabetes mellitus by changes in lifestyle among subjects with impaired glucose tolerance. The New England journal of medicine. 2001; 344(18):134350 .

\begin{tabular}{|c|c|c|c|}
\hline \multicolumn{4}{|c|}{ AUTHORSHIP AND CONTRIBUTION DECLARATION } \\
\hline Sr. \# & Author(s) Full Name & Contribution to the paper & Author(s) Signature \\
\hline 1 & $\begin{array}{l}\text { Sarah Sadiq } \\
\text { Asifa Majeed }\end{array}$ & $\begin{array}{l}\text { Conception and design of } \\
\text { the work, data collection, } \\
\text { performance of research work, } \\
\text { drafting of article. } \\
\text { Design of work, SUpervision of } \\
\text { research and proof reading of } \\
\text { article. } \\
\text { Guidance through the } \\
\text { research techniques, analysis, } \\
\text { interpretation of data for the } \\
\text { work. }\end{array}$ & $\frac{4}{180}$ \\
\hline
\end{tabular}

